\title{
Antiradical capacities of noodle containing perilla leaf
}

\author{
H. E. Hyun, E. H. Lee, J. E. Seong and Y. O. Song \\ Department of Food and Nutrition and Kimchi Research Institute, Pusan National University, Busan 609-735, Korea
}

\begin{abstract}
Noodles are getting popular in the East Asia where the main meal is cooked rice ${ }^{(1)}$. In Korea, young generation prefers noodle rather than rice since preparation and serving of rice meal is time consuming. But the nutritional quality of noodle is comparable to that of rice meal. Recently there are demands for special noodles with bright colours on top of health promoting effects. The market for the functional noodle emphasised on colour is increasing. Fruits, vegetables and nuts such as green tea, pine needle and wild berry are added to the flour during the process of dough making. Among these, greenish colour noodles are preferred the most. The aim of this study is to examine the radical scavenging activity of noodle manufactured with perilla leaf (PL) whose colour is green. DPPH radical, hydroxyl radical and superoxide anion scavenging activity are determined. Flour noodle $(\mathrm{FN})$ is used as a control to compare the radical scavenging activity of flour noodle containing perilla leaf (FNPL). Radical scavenging activity of PL is also studied. For the study, $70 \%$ ethanol extracts were prepared. DPPH radical scavenging activity was determined by Hatano et $a l^{(2)}$. Superoxide anion scavenging activity was measured using the nitroblue tetrazolium (NBT) reduction method described by Robak $\mathrm{J}$ et al. $^{(3)}$. Hydroxyl radical scavenging activity was evaluated by the method of Nagai et al. ${ }^{(4)}$.
\end{abstract}

Table 1. $\mathrm{IC}_{50}$ for DPPH radical scavenging activity $(\%)$

\begin{tabular}{lr}
\hline & IC50 (mg/ml) \\
\hline PL & $0.05^{*}$ \\
FNPL & $0.56^{*}$ \\
FN & $11.05 \dagger$ \\
\hline$* \dagger$ Data with different letters in the row are significantly \\
\multicolumn{2}{l}{ different (ANOVA, followed by Duncan's multiple range } \\
test), $P<0.05$.
\end{tabular}

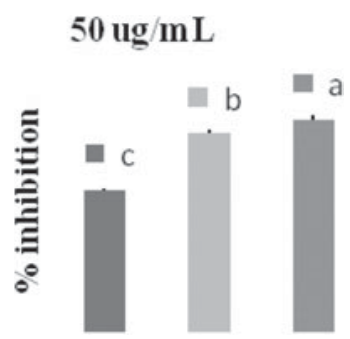

Fig. 1. Hydroxyl radical scavenging activity (\%). ${ }^{\mathrm{a}-\mathrm{c}}$ Data with different letters are significantly different with $P<0.05$ (ANOVA, followed by Duncan's test).
$3 \mathrm{mg} / \mathrm{mL}$

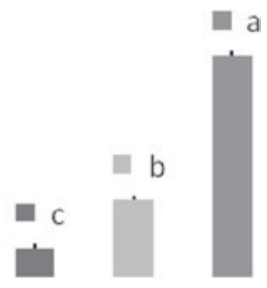

Fig. 2. Superoxide anion radical scavenging activity $(\%)$

As shown in Table $1, \mathrm{IC}_{50}$ for DPPH radical for the FNPL is 20 -folds higher than that of FN. This result inform us that addition of PL $(15 \%)$ in the flour noodle increased radical scavenging activity significantly compared with the plain noodle $(P<0.05)$. This effect was confirmed in the experiments of hydroxyl radical and superoxide anion radical scavenging activity. As shown in Figs. 1 and 2, radical scavenging activities of FNPL were higher than those of FN. According to the results from the sensory evaluation, the preference for FNPL was superior to FN, especially for the colour and taste. In conclusion, people consumes the flour noodle containing perilla leaf would increase their anti-oxidative capacity on top of enjoying the colour of noodle.

This work was supported by a grant from the Regional Innovation System (RIS) Program by the Ministry of Commerce, Industry \& Energy, Republic of Korea.

1. Yang HS, Kim CS (2010) J Korean Soc Food Sci Nutr 39, 737-744.

2. Hatano T, Edamatsu R, Hiramatsu M et al. (1989) Chem Pharm Bull 37, 2016-2021.

3. Robak J, Gryglewski RJ (1988) Biochem Pharmacol 37, 837-841.

4. Nagai T, Inoue R et al. (2002) J Nutr Res 22, 519-526. 